\title{
Optofluidic applications with lithium niobate nanowires
}

\author{
Rachel Grange ${ }^{\mathrm{a}}$, Jae-Woo Choi ${ }^{\mathrm{a}, \mathrm{b}}$ Chia-Lung Hsieh ${ }^{\mathrm{a}, \mathrm{b}}, \mathrm{Ye} \mathrm{Pu}^{\mathrm{a}}$, and Demetri Psaltis*a \\ ${ }^{a}$ EPFL, School of Engineering, Station 17, 1015 Lausanne, Switzerland; \\ ${ }^{\mathrm{b}}$ Department of Electrical Engineering, California Institute of Technology, 1200 East \\ California Boulevard, MC 136-93, Pasadena, California 91125, USA
}

\begin{abstract}
We report the hydrothermal synthesis of free-standing lithium niobate nanowires. We show that the versatile properties of bulk lithium niobate such as nonlinear optical effects can be exploited at the nanoscale. We describe the fabrication of polydimethylsiloxane (PDMS) microfluidics as well as indium tin oxide (ITO) electrodes with different design for dedicated applications. The control of microfluidic channel dimensions and the corresponding particle concentration is explored. Finally, the selection of fluidic conductivity for optimal dielectrophoretic trapping conditions is discussed.
\end{abstract}

Keywords: Lithium niobate, nanowire, second-harmonic generation, hydrothermal synthesis, polarization.

\section{INTRODUCTION}

Bulk lithium niobate $\left(\mathrm{LiNbO}_{3}\right)$ is an optical and electronic material that shows photovoltaic, piezoelectric, ferroelectric, photorefractive and nonlinear optical effects. The photovoltaic effect from bulk $\mathrm{LiNbO}_{3}$, generating an electrical dipole has for instance been used for the dielectrophoretic trapping of particles ${ }^{1}$. Similarly the piezoelectric effect was utilized for dieletrophoretic trapping of microparticles ${ }^{2}$. Nanowire syntheses have enabled further device miniaturization to the nanoscale. Furthermore, the utilization of microfluidics has enabled several interesting optofluidic applications.

Many synthesis routes are being developed for $\mathrm{LiNbO}_{3}$ at a nanoscale resulting in different size, shape and crystalline quality (Table 1). $\mathrm{LiNbO}_{3}$ nanoparticles have been previously produced by milling ${ }^{3}$, nonaqueous route ${ }^{4}$, sol-gel method ${ }^{5}$, or hydrothermal route ${ }^{6}$. Array of polycrystalline $\mathrm{LiNbO}_{3}$ nanotubes have also been reported ${ }^{7}$, as well as a solution-phase synthesis to produce rod-like structures among other multiple structures ${ }^{8}$. We demonstrate the first synthesis of freestanding nanowire ${ }^{9}$. The utility of such nanowires is to take advantage of their field gradients for biosensing applications or self assembly ${ }^{10}$.

Table 1. Synthesis routes followed so far for one dimensional nanoscale lithium niobate.

\begin{tabular}{|l|l|l|}
\hline Ref. & Synthesis process and advantages & Particle shape and size \\
\hline${ }^{3}$ & High-energy ball milling & $10-25 \mathrm{~nm}$ crystals \\
\hline 5 & Nonaqueous route and low temperature $\left(200-200^{\circ} \mathrm{C}\right)$ & $20-50 \mathrm{~nm}$ \\
\hline 6 & $\begin{array}{l}\text { Sol-gel treatment of alkoxides, lower preparation } \\
\text { temperature than solid-state reactions }\left(>1200^{\circ} \mathrm{C}\right)\end{array}$ & $40-60 \mathrm{~nm}$, with heat treatment at $600^{\circ} \mathrm{C}$ \\
\hline 7 & $\begin{array}{l}\text { Hydrothermal route, } 250^{\circ} \mathrm{C} \text { autoclave, low temperature, } \\
\text { high yields possible for industrial applications }\end{array}$ & Flakes like shape of $40-100 \mathrm{~nm}$ \\
\hline 8 & $\begin{array}{l}\text { Array of nanotubes through porous alumina templates, } \\
\text { possibility to create specifically patterned arrays of 1D } \\
\text { structures }\end{array}$ & $\begin{array}{l}\text { Nanotubes with diameters from a few tens to a few } \\
\text { hundreds of nanometers and a wall thickness on the } \\
\text { order of 20 nm. }\end{array}$ \\
\hline $\begin{array}{l}\text { This } \\
\text { wolution-phase, easy to tune the material composition }\end{array}$ & $\begin{array}{l}\text { Anisotropic structures with lengths up to } \\
100 \text { nm and diameters around } 7 \mathrm{~nm} .\end{array}$ \\
\hline
\end{tabular}

*demetri.psaltis@epfl.ch; phone +41 21693 7795; fax +41 21693 6930; http://lo.epfl.ch

Active Photonic Materials III, edited by Ganapathi S. Subramania, Stavroula Foteinopoulou,

Proc. of SPIE Vol. $7756,77560 \mathrm{H} \cdot$ C 2010 SPIE · CCC code: $0277-786 X / 10 / \$ 18 \cdot$ doi: $10.1117 / 12.860401$ 
With lithium niobate nanowires, we have previously demonstrated the capability to optically generate electric fields. These fields were used to modulate birefrigent media such as liquid crystals or E. coli ${ }^{11}$. The optically controlled liquid crystals could be used as display or lens elements. The E. coli aligned to the electric fields generated by the nanowires. In this study, we will explore the possibility of aligning and trapping the nanowires through the use of dielectrophoresis and electro-orientation. First, the parameters of synthesis will be given.

In this study, we will explore the possibility of aligning and trapping the nanowires through the use of dielectrophoresis and electro-orientation. First, the parameters of synthesis will be given. Next, the fabrication of polydimethylsiloxane (PDMS) microfluidic devices with indium tin oxide (ITO) electrodes will be discussed. Also, the control of the microfluidic channel dimensions and the corresponding particle concentration will be explored. Finally, the selection of fluidic conductivity for optimal dielectrophoretic trapping conditions will be explained. In summary, our experimental results will be shown as well as a brief discussion regarding directed assembly will take place.

\section{SYNTHESIS OF LITHIUM NIOBATE NANOWIRES}

The $\mathrm{LiNbO}_{3}$ nanowires are synthesized using the hydrothermal route at low temperatures, following the synthesis of $\mathrm{KNbO}_{3}$ nanowires ${ }^{12}$. Niobium pentoxide $\left(\mathrm{Nb}_{2} \mathrm{O}_{5}\right)$ powder is added to distilled water in which lithium hydroxide powder $(\mathrm{LiOH})$ is dissolved. The mixture (in a Teflon vessel) is heated in an autoclave at about $150^{\circ} \mathrm{C}$ and under a pressure of about 5 bars during 6 days, producing a precipitate. The solid precipitate is filtered, washed with water and ethanol, and dried at $120^{\circ} \mathrm{C}$. The X-ray diffraction pattern of the synthesized $\mathrm{LiNbO}_{3}$ powder correlates with the peak positions of standard bulk $\mathrm{LiNbO}_{3}$ (Figure $1 \mathrm{a}$ ). Before preparing the transmission electron microscope (TEM) and the scanning electron microscope (SEM) samples, the lithium niobate precipitate is suspended in methanol to have a fast drying process and avoid aggregates of nanowires on substrates. TEM and SEM pictures show typical nanowires up to few $\mu \mathrm{m}$ long and around $50 \mathrm{~nm}$ in diameter (Figure $1 \mathrm{~b}$ and c). The fact that free-standing nanowires are observed is probably due to the low solubility of $\mathrm{Nb}_{2} \mathrm{O}_{5}$ during the liquid phase, which is instantaneously used to form $\mathrm{LiNbO}_{3}{ }^{13}$.

(a)

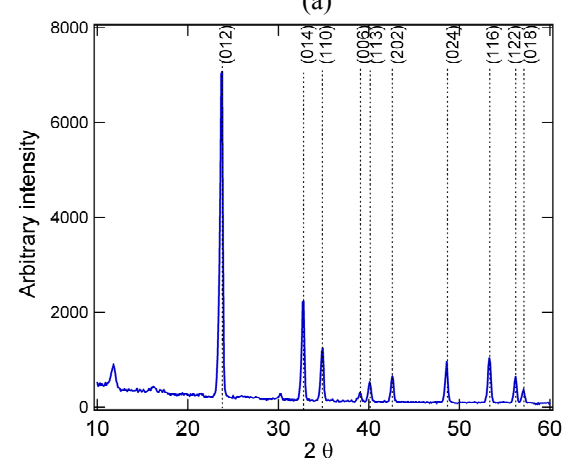

(b)

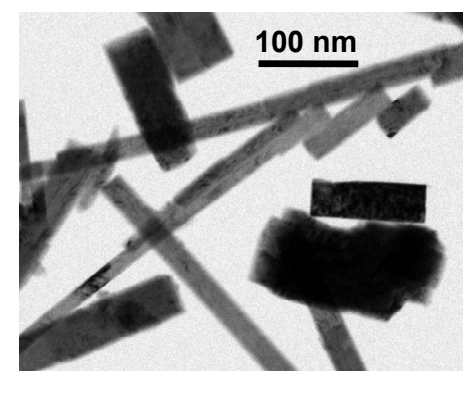

(c)

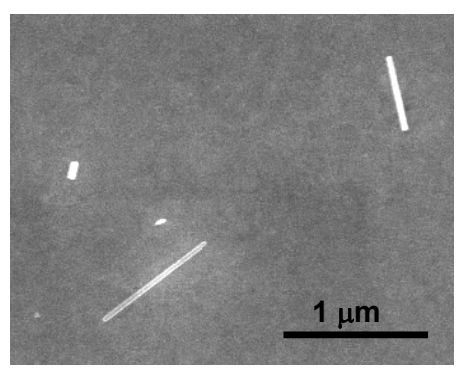

Figure 1. (a) X-ray diffraction pattern of $\mathrm{LiNbO}_{3}$ synthesized nanopowder. The dashed lines indicate the location of standard bulk $\mathrm{LiNbO}_{3}$ peaks. (b) TEM image. (c) SEM image. 


\section{DEVICE FABRICATION}

The optofluidics device fabrication implies different process flows depending on the applications (Figure 2). In case of microfluidic channels that are made of polydimethylsiloxane (PDMS), the fabrication uses the replica molding technique (Figure 2 a). A master mold is produced through UV lithography with SU-8 (GM-1070, Gerstelec) on a silicon wafer. After trimethylchlorosilane (Sigma Aldrich) treatment of the master mold for $5 \mathrm{~min}$, the PDMS (Sylgard 184, Dow Corning) is poured onto the mold with a $10: 1$ base-to-curing agent ratio. After curing in an oven at $80^{\circ} \mathrm{C}$ for $1 \mathrm{~h}$, the silicone is released from the mold.

For dielectrophoresis applications, a $1 \mathrm{~mm}$ thick glass plate with a conductive electrode pattern in indium tin oxide (ITO) is used as described in ${ }^{14}$. The sample is fabricated using contact photo lithography (Figure $2 \mathrm{~b}$ ). First, we obtain ITO coated glass plates (Aldrich) rated at 30-60 $\Omega$ /square. Next, positive photoresist (S1813) is spincoated onto the plates. A pattern is exposed using a UV mask aligner and developed. Finally, the ITO is etched to create the electrode pattern.

\section{(a) PDMS process flow}

1. Silicon wafer

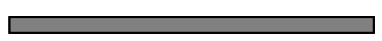

2. Spincoat photoresist

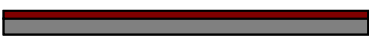

3. UV exposure

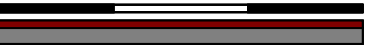

4. Bake

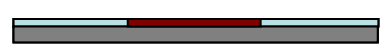

5. Develop

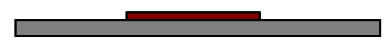

6. Surface treatment with TMCS

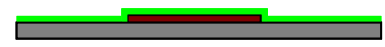

7. Pour PDMS and bake

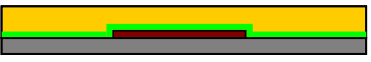

(b) ITO etch process flow

2. Spincoat photoresist

3. UV exposure

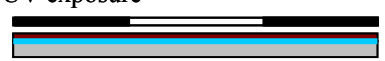

4. Bake

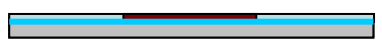

5. Develop

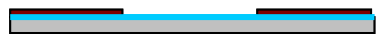

6. ITO Etch

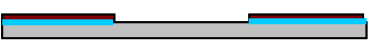

7. Resist removal

Figure 2. (a) PDMS process flow, (b) ITO process flow

For the first type of device, a floating dielectrophoresis electrode design was utilized to prevent shorting the nanowire after being trapped ${ }^{15}$. This concept is best demonstrated by the COMSOL simulation (Figure 3). A voltage is placed between the top and bottom electrodes. Due to the geometry of the floating electrodes, high electric fields are generated at the tips in the middle floating electrodes. The direction and magnitude of the electric field is indicated by the arrows. As will be discussed in the section 4, the high electric fields will serve as dielectrophoretic traps and the direction of the electric field will align the nanowires due to electro-orientation. For the specific design, triangular ITO electrodes were designed with a 2 micron gap and the triangular ITO electrodes were separated by the contact electrodes with a 5 micron gap. 


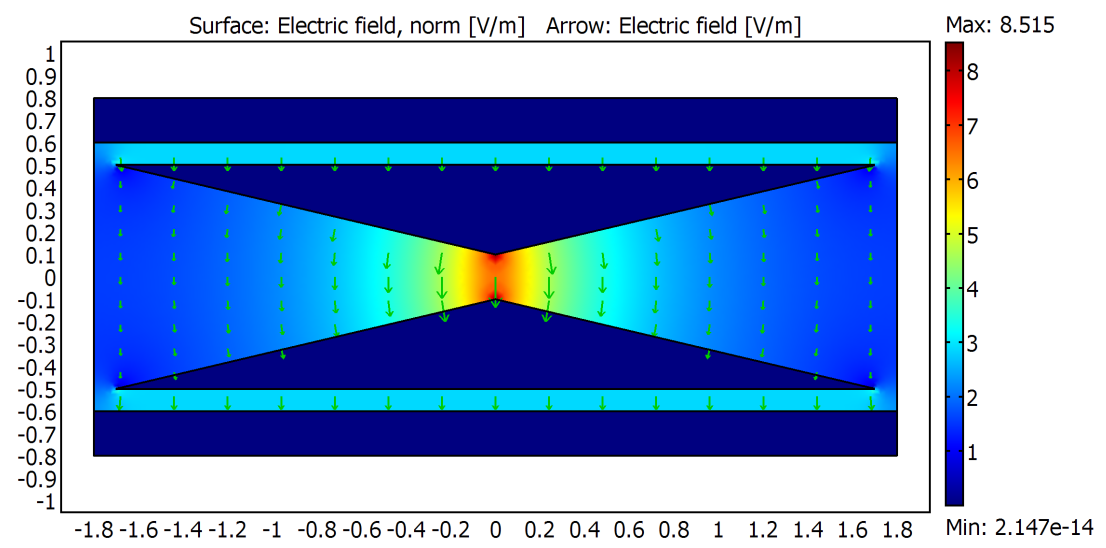

Figure 3: COMSOL simulation of the electrode design

A standard ITO etch with a 5:6:1 ratio of $\mathrm{H}_{2} \mathrm{O}: \mathrm{HCl}(40 \%): \mathrm{HNO}_{3}(60 \%)$ at room temperature was utilized. By changing the etching time, we were able to control the size of the gap between the triangular electrodes. With a 5 minute etch, the electrodes were shorted and there was no gap. At 10 minutes, a 1 micron gap existed. By 15 minutes, a 2 micron gap was formed. Results utilized a 10 to 15 minute electrode etch.

A PDMS microfluidic channel with the dimensions of 100 micron wide, 10 micron deep and $1.5 \mathrm{~cm}$ long was placed to overlap with the electrode gap. The microfluidic channel can hold $15 \mathrm{~nL}$ of liquid. To obtain at least a single nanowire in the entire channel, a concentration of $6.7 \times 10^{4}$ particle/mL is required. Figure 4 shows the trapping of several nanowires with the floating electrodes design.

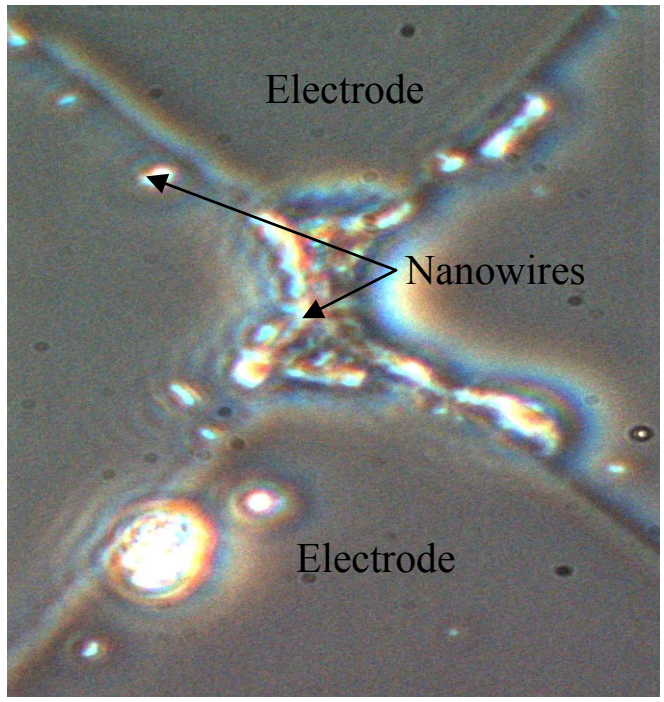

Figure 4: White light image of the trapping of Lithium niobate nanowires at the tips of the floating electrodes 


\section{DIELECTROPHORESIS AND ELECTROORIENTATION RESULTS}

The second device we developed was to manipulate lithium niobate nanowires in a fluidic environment and monitor the second-harmonic generation (SHG) response using an optical trapping setup. The conductive electrode pattern is interdigitated with specific spacing and width that were previously determined ${ }^{14}$. The optical tweezer is generated with a specific polarization that is determined with a half wave plate and located outside of the fluidic region, close to either substrate. Therefore, the nanowire is not allowed to orient along the direction of propagation and it locates itself orthogonal to the beam ${ }^{16}$. This polarization imparts a force to orient the nanowire. Futhermore, an external electric field is applied to the nanowires using the pattern of electrodes described in section 3. This external electric field induces an additional electro-orientation force caused by the dielectrophoretic (DEP) response of the $\mathrm{LiNbO}_{3}$ nanowires. Note that the torque on the nanowire due to the external electric field is almost ten times greater in magnitude than the torque on the nanowire due to the optical polarization of the laser for the particle orientation denoted above.

Figure 5 shows a sequence of white light images of a nanowire suspended in deionised water. At time $\mathrm{t}=0$, no electric field $\left(\mathrm{E}_{\text {electrodes }}\right)$ is applied and the nanowire is only tweezed by the laser $\left(\mathrm{E}_{\text {laser }}\right)$ with a polarization parallel to the electrodes. Then a $10 \mathrm{~V}_{\mathrm{pp}}$ electric field with a frequency of $150 \mathrm{kHz}$ is applied and after 1 second, the wire is oriented along this electric field due to the stronger field than the one from the laser.

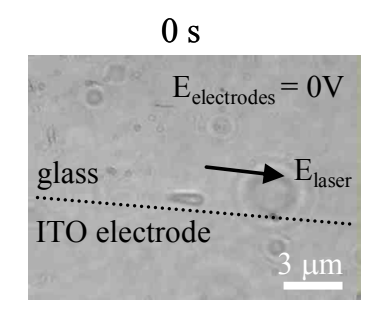

$0.334 \mathrm{~s}$

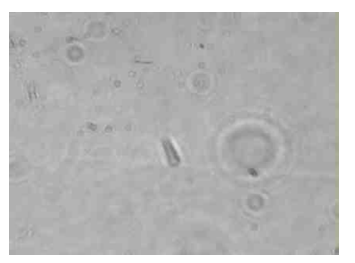

$0.033 \mathrm{~s}$

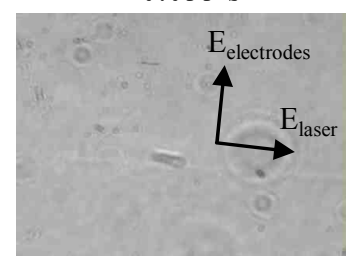

$0.901 \mathrm{~s}$

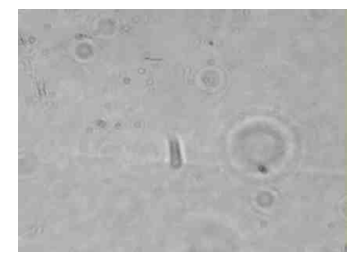

$0.200 \mathrm{~s}$

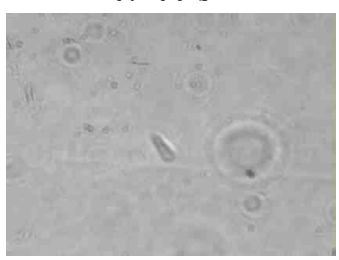

$1.001 \mathrm{~s}$

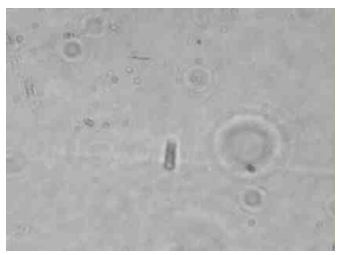

Figure 5. White light images sequence of DEP response of a $\mathrm{LiNbO}_{3}$ nanowire. The nanowire was suspended in deionized water and an electric field of $10 \mathrm{~V}_{\mathrm{pp}}$ at $150 \mathrm{kHz}$ was applied.

Figure 6 shows the SHG response measured with and without the applied electric field. The polarization dependency of the nanowire allows for the detection its position. The SHG signal can then be useful for detecting smaller wire close to the diffraction limit of a bright field microscope.
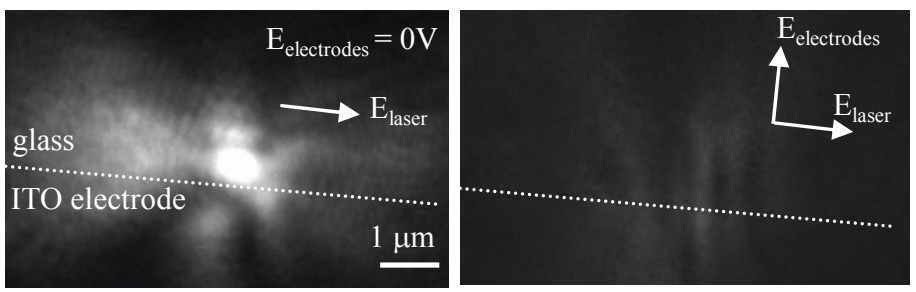

Figure 6. SHG response of the $\mathrm{LiNbO}_{3}$ nanowire. (Left) Laser field only. (Right) Laser and electrodes field.

The $\mathrm{LiNbO}_{3}$ nanowires are aligned with the field due to positive DEP forces. Increasing the conductivity of the suspension as well as changing the frequency of the applied electric field can change the type of DEP force on the nanowire which will result in the nanowire to not be aligned with the field. ${ }^{17}$ The change in DEP force, as the 
conductivity and frequency of electric field is applied, is demonstrated in Table 2 . We notice the crossover conductivity between negative and positive DEP when the conductivity of the solution is around $170 \mu \mathrm{S} / \mathrm{cm}$.

Table 2. Change in DEP force at different conductivity and frequency of electric field.

\begin{tabular}{|l|l|l|}
\hline Conductivity & Electric field frequency $\mathbf{1 0 0} \mathbf{k H z}$ & Electric field frequency $\mathbf{1 ~ M H z}$ \\
\hline $0 \mu \mathrm{S} / \mathrm{cm}$ (Deionized water) & Positive DEP response & Positive DEP response \\
\hline$<60 \mu \mathrm{S} / \mathrm{cm}$ & Positive DEP response & Positive DEP response \\
\hline $170 \mu \mathrm{S} / \mathrm{cm}$ & Negative DEP response & Positive DEP response \\
\hline$>300 \mu \mathrm{S} / \mathrm{cm}$ & Negative DEP response & Negative DEP response \\
\hline
\end{tabular}

DEP forces have been utilized to orient and manipulate several different types of dielectric nanowires. ${ }^{18}$ The laser beam in the optical tweezer setup helps locate the nanowire, especially when the particle is sub-wavelength in size and cannot be optically resolved, through both its trapping ability and the capability of generating SHG. The combination provided by the applied external electric field and the polarization of the laser beam serves useful for several measurements in the nanometer scale. For instance, an estimation of the conductivity near the nanowire may be obtained. This may be useful if there is a conductivity gradient in the sample ${ }^{19}$.

\section{CONCLUSION}

We report the synthesis of free-standing lithium niobate nanowires following the hydrothermal route. The length can be several micrometers for a width of about 50 to $70 \mathrm{~nm}$. Then, we demonstrated the optical and electrical manipulation of the nanowire in a fluidic environment. We tested two kinds of electrode designs. First of all, we demonstrate the trapping of many nanowires with floating electrodes. Then, we also showed the laser tweezing and change in orientation of nanowires with an electric field while monitoring the SHG signal.

\section{ACKNOWLEDGEMENTS}

The authors like to thank L. Forro's group at EPFL for the synthesis of the lithium niobate nanowires.

\section{REFERENCES}

[1] Eggert, H. A., Kuhnert, F. Y., Buse, K., Adleman, J. R., and Psaltis, D., "Trapping of dielectric particles with light-induced space-charge fields," Applied Physics Letters,90 (2007).

[2] Grilli, S. and Ferraro, P., "Dielectrophoretic trapping of suspended particles by selective pyroelectric effect in lithium niobate crystals," Applied Physics Letters,92,232902-232903 (2008).

[3] Schwesyg, J. R., Eggert, H. A., Buse, K., Sliwinska, E., Khalil, S., Kaiser, M., and Meerholz, K., "Fabrication and optical characterization of stable suspensions of iron- or copper-doped lithium niobate nanocrystals in heptane," Applied Physics B-Lasers and Optics,89,15-17 (2007).

[4] Niederberger, M., Pinna, N., Polleux, J., and Antonietti, M., "A general soft-chemistry route to perovskites and related materials: Synthesis of BaTiO3, BaZrO3, and LiNbO3 nanoparticles," Angewandte ChemieInternational Edition, 43,2270-2273 (2004).

[5] Wang, L. H., Yuan, D. R., Duan, X. L., Wang, X. Q., and Yu, F. P., "Synthesis and characterization of fine lithium niobate powders by sol-gel method," Crystal Research and Technology,42,321-324 (2007).

[6] An, C. H., Tang, K. B., Wang, C. R., Shen, G. Z., Jin, Y., and Qian, Y. T., "Characterization of LiNbO3 nanocrystals prepared via a convenient hydrothermal route," Materials Research Bulletin,37,1791-1796 (2002).

[7] Zhao, L. L., Steinhart, M., Yosef, M., Lee, S. K., and Schlecht, S., "Large-scale template-assisted growth of $\mathrm{LiNbO} 3$ one-dimensional nanostructures for nano-sensors," Sensors and Actuators B-Chemical,109,86-90 (2005).

[8] Wood, B. D., Mocanu, V., and Gates, B. D., "Solution-Phase Synthesis of Crystalline Lithium Niobate Nanostructures," Advanced Materials,20,4552-4556 (2008). 
[9] Grange, R., Choi , J.-W., Hsieh, C.-L., Pu, Y., Magrez, A., Smadja, R., Forro, L., and Psaltis, D., "Lithium niobate nanowires synthesis, optical properties, and manipulation," Applied Physics Letters,95,143105 (2009).

[10] Grzelczak, M., Vermant, J., Furst, E. M., and Liz-Marzan, L. M., "Directed Self-Assembly of Nanoparticles," ACS Nano (2010).

[11] Pethig, R. and Markx, G. H., "Applications of dielectrophoresis in biotechnology," Trends in Biotechnology, 15,426-432 (1997).

[12] Magrez, A., Vasco, E., Seo, J. W., Dieker, C., Setter, N., and Forro, L., "Growth of single-crystalline KNbO3 nanostructures," Journal of Physical Chemistry B,110,58-61 (2006).

[13] Vasco, E., Magrez, A., Forro, L., and Setter, N., "Growth kinetics of one-dimensional KNbO3 nanostructures by hydrothermal processing routes," Journal of Physical Chemistry B,109,14331-14334 (2005).

[14] Choi, J. W., Pu, A., and Psaltis, D., "Optical detection of asymmetric bacteria utilizing electro orientation," Optics Express, 14,9780-9785 (2006).

[15] Banerjee, S., White, B., Huang, L., Rego, B. J., O'Brien, S., and Herman, I. P., "Precise positioning of carbon nanotubes by ac dielectrophoresis using floating posts," Applied Physics a-Materials Science \& Processing,86,415-419 (2007).

[16] Nakayama, Y., Pauzauskie, P. J., Radenovic, A., Onorato, R. M., Saykally, R. J., Liphardt, J., and Yang, P. D., "Tunable nanowire nonlinear optical probe," Nature,447,1098-U1098 (2007).

[17] Green, N. G. and Morgan, H., "Dielectrophoretic separation of nano-particles," Journal of Physics D-Applied Physics,30,L41-L44 (1997).

[18] Burke, P. J., "Nano-dielectrophoresis: Electronic Nanotweezers," in Encyclopedia of Nanoscience and Nanotechnology, vol. 6, H. S. Nalwa, Ed., 2004, pp. 623.

[19] Markx, G. H., Dyda, P. A., and Pethig, R., "Dielectrophoretic separation of bacteria using a conductivity gradient," Journal of Biotechnology,51,175-180 (1996). 